This item was submitted to Loughborough's Institutional Repository (https://dspace.lboro.ac.uk/) by the author and is made available under the following Creative Commons Licence conditions.

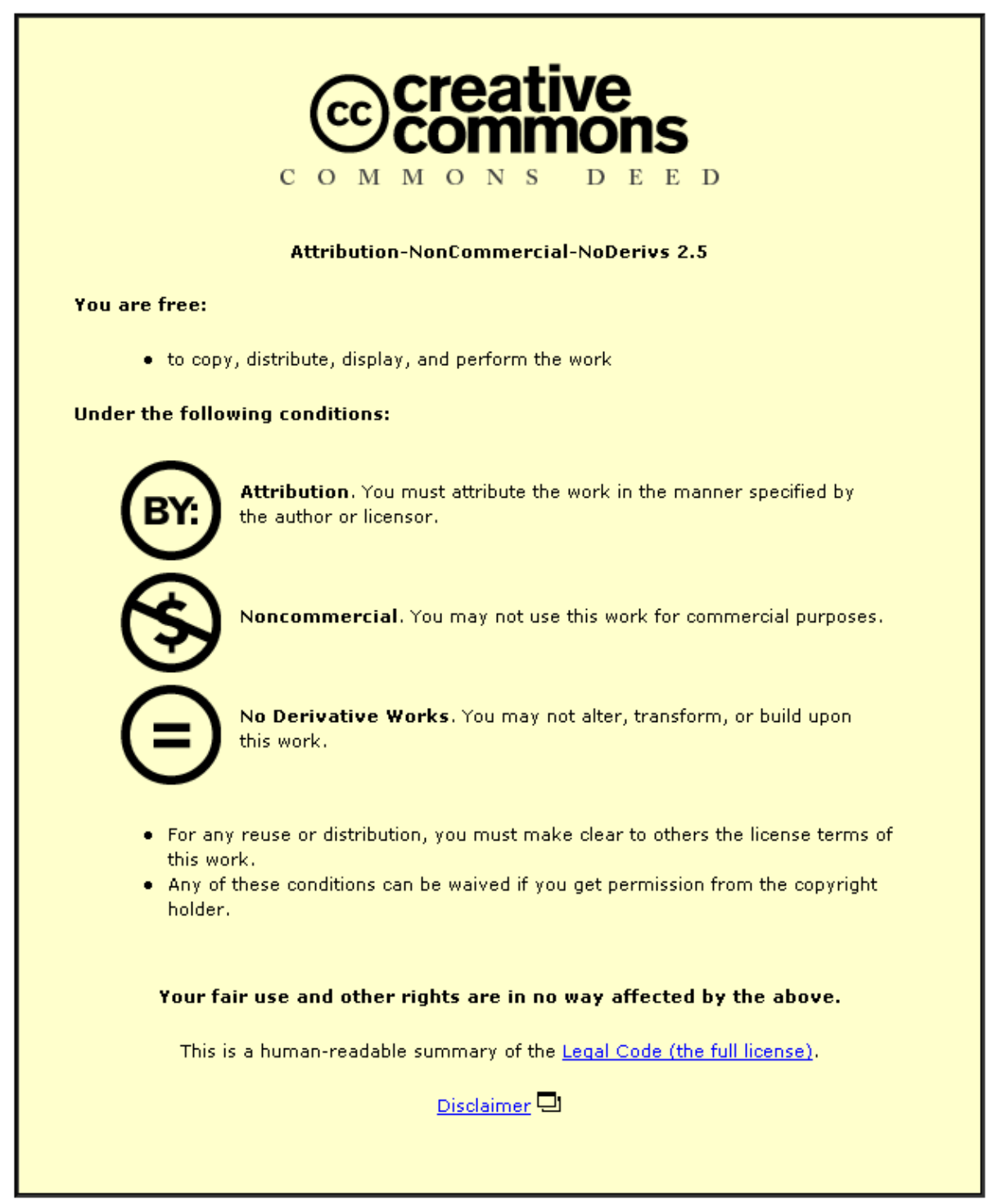

For the full text of this licence, please go to: http://creativecommons.org/licenses/by-nc-nd/2.5/ 


\title{
Modeling the Inactivation Kinetics of Bacillus subtilis Spores by Nonthermal Plasmas
}

\author{
Stefano Perni, Xutao T. Deng, Gilbert Shama, and Michael G. Kong, Senior Member, IEEE
}

\begin{abstract}
The inactivation performances of different nonthermal plasmas are often compared with each other in terms of their decimal reduction values, typically obtained by linearizing selected segments of their inactivation curves. However, this approach is subjective and can result in uncertainties in the prediction of this parameter. To overcome this, in this paper, the application of models capable of describing inactivation curves in their entirety is considered. The authors employ the Baranyi and Weibull models, both commonly used for microbial inactivation by heat. An empirical model based on a third-order polynomial to seek greater accuracy is further proposed. Using these three inactivation models, predictions of decimal reduction values for 11 plasma inactivation studies of Bacillus subtilis spores are obtained and compared with their reported values. Although the agreement obtained between these different approaches is generally fair, the current practice of segmented linearization is shown to be overly simplistic. A rigorous model is therefore critical to capture the essentially nonlinear character of plasma inactivation kinetics and hence allow for an objective comparison of the performances of similar biocidal nonthermal plasmas.
\end{abstract}

Index Terms-Bacillus subtilis spores, decimal reduction times, inactivation models, nonthermal plasmas.

\section{INTRODUCTION}

$\mathbf{T}$ HE THERMALLY induced inactivation of microorganisms is widely and routinely used to ensure the microbiological safety of a range of products. Although generally effective, conventional thermal processes are not suitable in instances where the object or product to be microbially decontaminated is heat sensitive and may become irreversibly affected or otherwise damaged by the treatment. There are numerous individual examples of this, but the fresh food industry presents a particular case in point. This sector of the food industry has grown rapidly in recent years, reflecting changes in eating and cooking habits in Western societies [1]. Along with the rise in consumption of these foods have come increases in the incidences of foodborne diseases [2], and reliable measures to ensure the safety of fresh and uncooked foods would contribute greatly to improvements in public health. Conventional thermal treatment of such food can bring about unacceptable changes to its textural, organoleptic, and nutrient status [3] and is therefore unsuitable. Recently, it has been shown that nonthermal gas

Manuscript received October 11, 2005; revised May 18, 2006. This work was supported in part by the Department of Health, U.K.

S. Perni and G. Shama are with the Department of Chemical Engineering, Loughborough University, Loughborough LE11 3TU, U.K. (e-mail: G.Shama@lboro.ac.uk).

X. T. Deng and M. G. Kong are with the Department of Electronic and Electrical Engineering, Loughborough University, Loughborough LE11 3TU, U.K.

Digital Object Identifier 10.1109/TPS.2006.878434

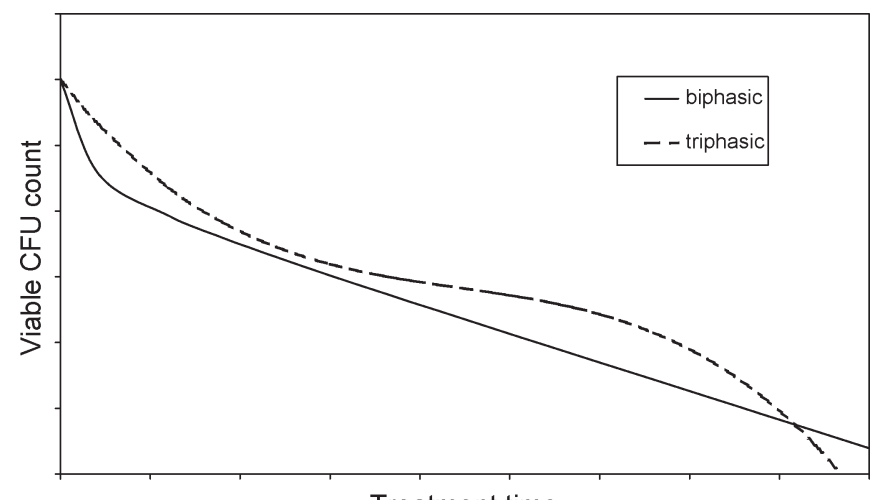

Fig. 1. Two common plasma inactivation kinetics that are sometimes described as termed biphasic or triphasic.

plasmas may be used to inactivate the microorganisms associated with fresh produce and present on the food surface in the form of "biofilms" (microorganisms embedded in an adhesive polysaccharide matrix) without affecting the physical appearance of the produce [4]. Therefore, nonthermal plasmas may have a significant role to play in the treatment of fresh and uncooked foods.

If nonthermal plasmas are to be adopted in food decontamination and other applications, it is essential that the kinetics of inactivation of a range of common pathogenic microorganisms are put on a quantitative basis and that the treatment times necessary to achieve a stated reduction in microbial viability are predictable with precision. Whereas a body of experimental data on the use of nonthermal plasmas for inactivating a diverse range of microorganisms is beginning to accumulate [4]-[6], the tools that have been used to analyze these data to date are unsophisticated and do not reflect the advances that have been made in modeling microbial inactivation [7]-[10]. The focal point for all such models is the microbial inactivation curve-a kinetic plot of cell viability against treatment time. Although plasma inactivation kinetics are essentially nonlinear [4]-[6], [11], the current approach has been to linearize selected segments of the microbial kinetics curve. This enables values of the decimal reduction time $D$-the time over which the treatment must be applied to achieve a reduction in the viability of the microbial population of $90 \%$ - to be obtained. This process of what might be termed "segmented linearization" has been extrapolated to provide evidence of a plurality of inactivation mechanisms and of the existence of different "inactivation phases" [12]. Fig. 1 shows two examples of common plasma inactivation curves that are commonly referred to by the terms "biphasic" and "triphasic." Without prescribing to the view that 
individual phases necessarily represent separate inactivation mechanisms, these terms will be used here for reference to this current view. Moreover, it is clear from Fig. 1 that plasma inactivation curves generally change in a smooth and continuous fashion without exhibiting distinct boundaries between adjacent phases [4]-[6], [11]. The identification of linear segments of inactivation curves is therefore inevitably subjective with the result that the $D$ values obtained are subject to uncertainty. In other words, the currently favored approach of segmented linearization for describing microbial inactivation using nonthermal plasmas is mathematically unreliable, and a more rigorous approach is therefore both necessary and desirable.

Microbial inactivation, by whatever means it is brought about, can in principle be analyzed by using empirical, mechanistic, or statistical models. Empirical models are simply mathematical equations that offer acceptable fits of the inactivation data. The segmented linearization method described above falls under this category. Mechanistic models are based to a greater or lesser extent on some concept of what effect the lethal treatment has on the cell or its individual components, whereas the statistical approach views the process of inactivation as being based on probabilities that individual cells within a population will be killed by the treatment in question. The latter two methodologies have been used to analyze microbial inactivation by thermal and other conventional methods [7]-[10] but have not been applied to the case of nonthermal plasma inactivation.

In this paper, we compare the fitting of the three fundamentally different types of inactivation model to both our own and previously published inactivation data for spores of the bacterium Bacillus subtilis by nonthermal plasmas, either generated in a vacuum chamber or in open air at atmospheric pressure. We employ the Baranyi model as a representative mechanistic model [7] and the Weibull model as a representative statistical model [9]. Both models have three parameters with which to fit experimental inactivation data. In an attempt to enable a better and more accurate fit, we propose an empirical model with four parameters based on a third-order polynomial function. The remainder of the paper is organized into an explanation - in Section II-of the theoretical basis of each of the three inactivation models used. In view of the fact that most authors seem to favor the quotation of $D$ values, we also present the derivation of this parameter from the individual model parameters. The application of all three models to the experimental data is presented in Section III, and our conclusions are given in Section IV.

\section{BASIS OF THE INACTIVATION MODELS}

A detailed description is presented here for each of the three inactivation models. The majority of inactivation curves obtained using nonthermal plasmas-whether in vacuum or at atmospheric pressure-are triphasic as earlier defined [4]-[6], [11]. Alternatively, their shape may be described as resembling an inverse-S shape. Notwithstanding similarities in form, it should not necessarily be inferred that the same, or even similar, inactivation mechanisms prevail when using different types of plasma systems. Whereas it is currently unclear whether this similarity in response stems from a commonality in the micro- bial target(s) affected by the various plasma species, it seems logical, in the first instance, to seek to develop inactivation models using data accumulated for a single species of organism. This approach should eliminate effects arising from differences in microbial structure between different species. In this paper, our focus is on spores of the bacterium B. subtilis for which there exists a large body of plasma inactivation data [11]-[14]. Each set of experimental data was fitted with the three models using DATAFIT 7.1 software (Oakdale Engineering, USA).

We chose to indicate the "goodness of fit" of all three models to the experimental data by recourse to the coefficient of multiple determination $\left(R^{2}\right)$, which is also known as the "Fisher" parameter [15]. This is defined as

$$
R^{2}=1-S / T
$$

where $S$ is the sum of the squared differences between values predicted by a model and its corresponding experimental data, and $T$ is the sum of the squared differences between the experimental data and the average value of all experimental data. A perfect fit is achieved when $R^{2}$ equals 1 .

In an attempt to correlate to decimal reduction values derived from the segmented linearization method, we employ the following definition of the $D$ value:

$$
N(t+D)=\frac{1}{10} N(t)
$$

where $N(t)$ is the cell concentration at time $t$.

\section{A. Baranyi Model}

The mechanistic model we used is based on the Baranyi inactivation model [7], which was itself developed from the widely used Baranyi-Roberts growth model [16]. Inactivation curves often display approximately horizontal sections-or "shoulders"- at low treatment times. Shoulders are those regions found at early treatment times and can be thought of as representing some threshold value of damage that must be exceeded before inactivation occurs. Such shoulders are modeled here by recourse to a so-called "critical component," denoted as $C_{c}$, which may or may not be a real substance present either inside or outside cells and, which, in this case, may be thought of as decaying in response to plasma treatment. Immediately after the shoulder section, the threshold for significant damage is reached, and the inactivation kinetics exhibit an exponential reduction in the number of viable cells. The Baranyi model describes this through an exponential function as detailed in (3). It can also account for the phenomenon of "tailing," where the inactivation curves essentially form a plateau at long treatment times. Tailing is generally a manifestation either of a heterogeneous population where some individual cells are more resistant than others or of physical phenomena such as cell clumping where those cells at the exterior of a clump effectively protect those at the interior. However, this is neglected in the form of the model that is used here and that was expressed by Greenacre et al. [8] as

$$
N(t)=N_{0} e^{-k_{\max } t}\left(\frac{1+C_{c}(0)}{1+C_{c}(0) e^{-k_{\max } t}}\right)
$$


where $N_{0}$ is the initial bacterial concentration, $k_{\max }$ is the maximum inactivation constant, and $C_{c}(0)$ is the initial concentration of the critical component $C_{c}$. Equation (3) has three fitting parameters (i.e., $N_{0}, C_{c}(0)$, and $\left.k_{\max }\right)$, and these will be numerically varied to achieve a best fit to a given set of experimental data. From a mechanistic point of view, these three parameters can be related directly to bacterial properties. With the definition of the decimal reduction time in (2), the above equation can be easily manipulated to yield the decimal reduction time at $t=0$ as follows:

$$
D_{1}=\frac{1}{k_{\max }} \ln \left[10+9 C_{c}(0)\right] .
$$

In some plasma inactivation studies, attempts have been made to describe the midsection of the inverse-S-shaped inactivation curve with a second decimal reduction value, or " $D_{2}$," as it is often referred to [12]. Mathematically, this can be derived by first determining the time at which the inflection occurs from

$$
\left.\frac{d^{2} N(t)}{d t^{2}}\right|_{t=t_{2}}=0 .
$$

Since $t=t_{2}$ occurs at the midpoint of the inflection section, or the midsection of the inverse $\mathrm{S}$, the value of $D_{2}$ is obtained from

$$
N\left(t_{2}+0.5 D_{2}\right)=\frac{1}{10} N\left(t_{2}-0.5 D_{2}\right) .
$$

A close examination of the Baranyi model in (3) suggests that it can never satisfy the condition imposed by (5). In other words, the Baranyi model may have too few parameters to allow for the estimate of $D_{2}$.

\section{B. Weibull Model}

The Weibull distribution function was originally formulated to predict the time to failure of mechanical components, but it has come to be widely applied to microbial inactivation by a variety of lethal agents. In this context, the model accounts for biological variation with respect to inactivation times. We take the following format of the model:

$$
N(t)=N_{0} 10^{-(t / \delta)^{p}}
$$

where the parameter $p$ is commonly referred to as the shape parameter, and $\delta$ is the time required-measured from $t=0$ - to reduce the viability of the cell population by $90 \%$. Therefore, $\delta$ is effectively the $D$ value at $t=0$, and this can readily be confirmed from (7). The shape parameter accounts for upward concavity of a survival curve $(p<1)$, a linear survival curve $(p=1)$, and downward concavity $(p>1)$. Although the model is essentially of an empirical nature, a link can be made with physiological effects. A value for $p<1$ indicates that the remaining cells have the ability to adapt to the applied stress, whereas $p>1$ indicates that the remaining cells become increasingly damaged [17]. Similar to the Baranyi model, the Weibull model has three fitting parameters, namely: 1) $N_{0}$, 2) $p$, and 3) $\delta$, and these can be varied until (7) achieves the best possible fit to any given set of experimental inactivation data. In practice, there is little room to vary $N_{0}$ since it is related to the initial bacterial density - a quantity that has an unambiguous physical meaning with a definitive value in any given experiment.

The decimal reduction value for the inflection section of the inactivation curve can again be derived using (5) and (6). Similarly to the Baranyi model, the Weibull model of (7) was found to be incapable of satisfying (5). It appears that the Weibull model has too few parameters to obtain a second decimal reduction value for the inflection section. It is worth mentioning that both the Baranyi and Weibull models have three fitting parameters and, therefore, their ability to achieve good fits of experimental data should be mathematically similar.

\section{Third-Order Polynomial-Based Empirical Model}

From a mathematical standpoint, the inverse-S shape of the plasma inactivation curve can be modeled empirically using polynomials. Since both the Baranyi and Weibull models have three parameters, it is useful to consider a third-order polynomial that can potentially provide four fitting parameters. Our empirical model takes the following general format:

$$
\log _{10} N(t)=y=a t^{3}+b t^{2}+c t+d .
$$

By setting $t=0$, one is left with

$$
d=\log _{10} N(0)=\log _{10} N_{0} .
$$

Using (2), (8), and (9), one obtains the decimal reduction value at $t=0$ that satisfies

$$
a D_{1}^{3}+b D_{1}^{2}+c D_{1}+1=0 .
$$

Although this expression can be solved analytically, it is in practice more convenient to solve it numerically after the four parameters, i.e., $a, b, c$, and $N_{0}$, are determined from a best fit of (8) to a given set of experimental data.

In contrast to the Baranyi and Weibull models, the empirical model of (8) can satisfy the conditions imposed by (5) and, thus, can be formulated to give the decimal reduction value for the inflection section of the inverse-S-shaped inactivation curve. Although not difficult to derive, the analytical expression of this second decimal reduction value is fairly cumbersome, and therefore, we calculated it numerically.

\section{RESUlts AND Discussion}

Table I shows the sources and details of 11 sets of experimental plasma inactivation data used to compare the three models and model predictions of the Fisher coefficient $R^{2}$ of (1). In general, all three models lead to a satisfactory fit to experimental data, with $R^{2}$ being above 0.95 for most cases and only falling below 0.85 on three occasions overall. This suggests that all three models reproduce plasma inactivation kinetics faithfully and reliably.

It is interesting to recall that the empirical model was introduced to provide additional fitting parameters than the other two models, so that it may offer a better fit to the experimental data. This is clearly the case for the second data set and is illustrated 
TABLE I

Details of 11 Plasma Inactivation Experiments and Their $R^{2}$ as Predicted With the Three Models

\begin{tabular}{|c|c|c|c|c|c|c|c|c|}
\hline \multirow{2}{*}{ Dataset } & \multicolumn{9}{|c|}{ Details of plasma inactivation conditions } & \multicolumn{3}{c|}{$R^{2}$ value } \\
\cline { 2 - 8 } & $\mathrm{Gas}$ & Gas pressure (torr) & $N_{0}$ & Shape of inactivation curve & Ref & Baranyi & Weibull & Empirical \\
\hline 1 & $\mathrm{Ar}$ & 7 & $1 \times 10^{6}$ & biphasic & {$[13]$} & 0.98 & 0.98 & 0.95 \\
\hline 2 & $\mathrm{Ar}+5 \% \mathrm{O}_{2}$ & 7 & $1 \times 10^{6}$ & triphasic & {$[13]$} & 0.83 & 0.82 & 0.98 \\
\hline 3 & $\mathrm{~N}_{2}+2 \% \mathrm{O}_{2}$ & 2 & $1 \times 10^{6}$ & biphasic & {$[13]$} & 0.96 & 0.95 & 0.98 \\
\hline 4 & $\mathrm{~N}_{2}+15 \% \mathrm{O}_{2}$ & 2 & $1 \times 10^{6}$ & triphasic & {$[13]$} & 0.94 & 0.95 & 0.99 \\
\hline 5 & $\mathrm{~N}_{2}$ & 5 & $1 \times 10^{6}$ & biphasic & {$[12]$} & 0.99 & 0.99 & 0.99 \\
\hline 6 & $\mathrm{~N}_{2}+0.7 \% \mathrm{O}_{2}$ & 5 & $1 \times 10^{6}$ & triphasic & {$[12]$} & 0.96 & 0.96 & 0.97 \\
\hline 7 & $\mathrm{~N}_{2}+1.5 \% \mathrm{O}_{2}$ & 5 & $1 \times 10^{6}$ & triphasic & {$[12]$} & 0.97 & 0.96 & 0.99 \\
\hline 8 & $\mathrm{CO}_{2}$ & $0.01-0.1$ & $3 \times 10^{6}$ & biphasic & {$[14]$} & 0.97 & 0.97 & 0.86 \\
\hline 9 & $\mathrm{CO}_{2}$ & $0.01-0.1$ & $1 \times 10^{7}$ & biphasic & {$[14]$} & 0.98 & 0.97 & 0.89 \\
\hline $10^{*}$ & $\mathrm{He}$ & 760 & $5 \times 10^{7}$ & biphasic & {$[11]$} & 0.97 & 0.98 & 0.97 \\
\hline $11 \dagger$ & $\mathrm{He}$ & 760 & $5 \times 10^{7}$ & biphasic & {$[11]$} & 0.99 & 0.99 & 0.98 \\
\hline
\end{tabular}

* spores produced at $22^{\circ} \mathrm{C} \quad \dagger$ spores produced at $47^{\circ} \mathrm{C}$

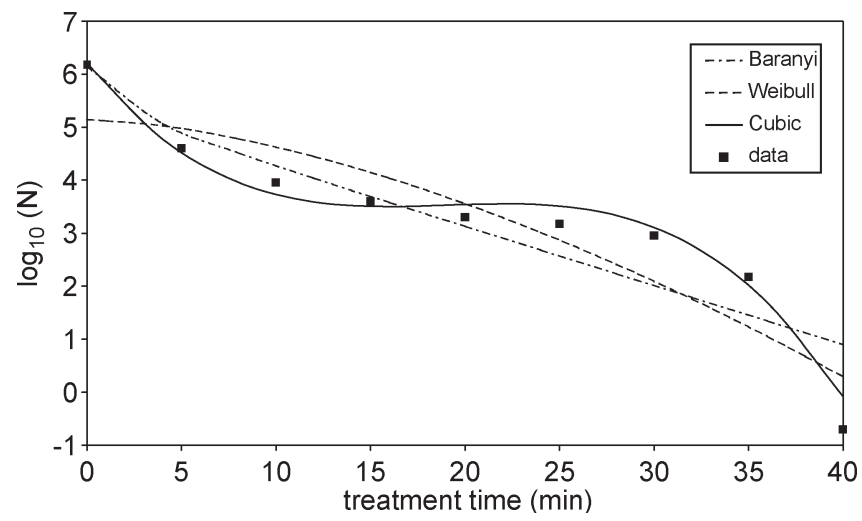

Fig. 2. Plasma inactivation data in the second data set and their modeling with all three proposed inactivation models.

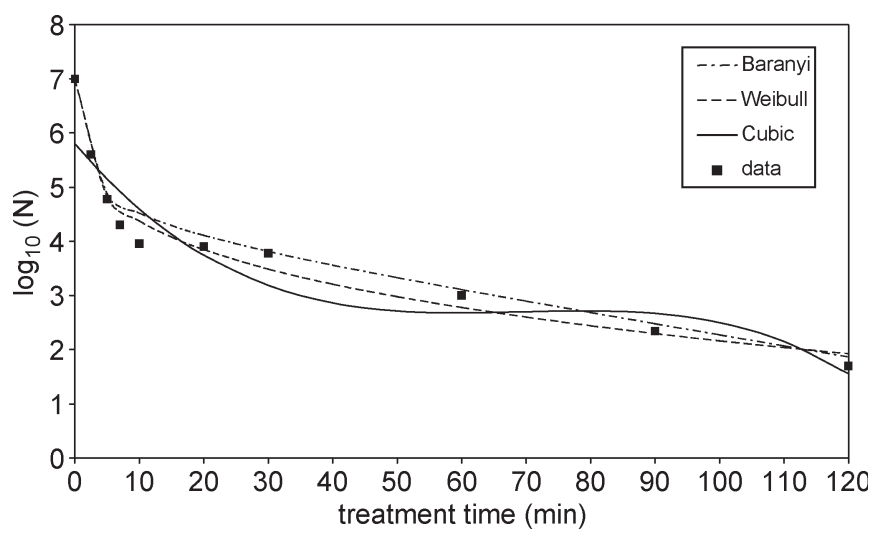

Fig. 3. Plasma inactivation data in the eighth data set and their modeling with all three proposed inactivation models.

in Fig. 2. Nevertheless, this is by no means always the case. For the eighth and ninth data sets, the fit by the empirical model is seen to be poorer than for the other two models, and this is shown in Fig. 3. Therefore, the additional parameter introduced in the empirical model of (8) does not necessarily lead to a more accurate representation of experimental inactivation data. For all other data sets, all three models result in similarly excellent fits to relevant experimental data. The overall conclusion is that all three inactivation models are similarly good.

A closer assessment of Table I reveals which inactivation model most closely fits each data set. It is intriguing to note that for inactivation curves not displaying a third phase, the fits achieved using the Baranyi and Weibull models are particularly good (the third, tenth, and 11th data sets) and, in some cases, even better than the polynomial equation (first, eighth, and ninth data sets). Van Boekel anticipated difficulties in modeling the thermal inactivation of spores with the Weibull model [17]. This was because heat both stimulates spore outgrowth and ultimately results in spore inactivation. However, thermal effects were insignificant in all cases considered in this paper, and our results show that for nonthermal microbial inactivation, the Weibull model is, on the whole, very adequate. Our findings therefore extend the application of the Weibull model to the description of microbial inactivation by nonthermal plasmas alongside those of other nonthermal inactivation techniques such as pulsed electric fields, ionizing radiation, and highpressure gases [17]. Equally, it is interesting to note that when the empirical model is applied to curves displaying a third phase (the second, fourth, sixth, and seventh data sets), the fitting is markedly improved, suggesting that, in these cases, the new approach presented here is mathematically better suited for dealing with this kind of inactivation curves as illustrated in Fig. 1 for the second data set.

Given that plasma inactivation kinetic curves are characteristically continuous and free of distinct boundaries between supposedly different inactivation phases, use of either of the three inactivation models is mathematically justified over the entire range of plasma treatment times. From a practical standpoint, it is interesting to establish whether the three models can be simplified to provide a single-parameter yardstick for characterizing and comparing different plasma inactivation curves. Segmented linearization with its predicted decimal reduction time provides one such yardstick, albeit a questionable one; where the inactivation curves are biphasic, two values of $D$ are quoted, and where they are triphasic, three values of $D$ are given. To evaluate how reliable, both quantitatively and qualitatively, this commonly used yardstick is, we calculate $D$ values using the three models and compare the predictions with values cited in the literature.

Table II summarizes the decimal reduction values of the first phase, $D_{1}$, predicted using all three models and also reported previously using segmented linearization. In general, the empirical third-order polynomial model offers the closest predictions to those obtained using segmented linearization, whereas the 
TABLE II

COMPARISON OF EXPERIMENTAL AND PREDicted $D$ VALUES

\begin{tabular}{|c|c|c|c|c|c|c|}
\hline \multirow{2}{*}{ Dataset } & \multicolumn{3}{|c|}{ Experimental D (min) } & \multicolumn{3}{|c|}{ Predicted D (min) } \\
\hline & $D_{1}$ & $D_{2}$ & $D_{3}$ & Baranyi & Weibull & Empirical \\
\hline 1 & 4 & 30 & N/A & 3.7 & 3.5 & 6.8 \\
\hline 2 & 2.9 & 23 & 1.7 & 3.5 & 15 & 2.5 \\
\hline 3 & 3.6 & 26 & N/A & 2.6 & 2.4 & 4.6 \\
\hline 4 & 3.6 & 26 & 3.5 & 6.9 & 16.8 & 5.5 \\
\hline 5 & not given & 45 & N/A & 37.1 & 36.9 & 37.6 \\
\hline 6 & 2.3 & 10.3 & 1.6 & 0.74 & 1.6 & 2.1 \\
\hline 7 & 2.3 & 10.3 & 1.8 & 1.7 & 4.03 & 2.6 \\
\hline 8 & $3-6$ & 40 & N/A & 0.38 & 0.27 & 8.1 \\
\hline 9 & $3-6$ & 40 & N/A & 1.7 & 2.08 & 13.9 \\
\hline 10 & 0.6 & \multirow{2}{*}{\multicolumn{2}{|c|}{ not given }} & 0.02 & 0.21 & 0.66 \\
\hline 11 & 1.05 & & & 0.84 & 0.99 & 1.22 \\
\hline
\end{tabular}

values yielded by the Weibull model show the greatest discrepancies. In particular, predictions of the empirical model agree well with those of the segmented linearization technique for triphasic inactivation curves (e.g., second, fourth, sixth, and seventh data sets) and for atmospheric plasmas (e.g., tenth and 11th data sets). On the other hand, the Baranyi and Weibull models are generally in good agreement with segmented linearization. There are, however, exceptions, in particular, the eighth and ninth data sets, for which all three models predict markedly different $D_{1}$ values to those obtained by segmented linearization. This may be partly due to a somewhat approximate nature in the prediction of the $D$ value in the original plasma inactivation study [14].

As was explained in Section II, the Baranyi and Weibull models do not support a mathematical derivation of $D$ values for the second and subsequent phases of the inactivation kinetics curve. The empirical model is, however, capable of predicting the second and third decimal reduction values. For all 11 cases in Table II, its predictions were found to be at variance with those obtained by segmented linearization method and, thus, are not included in Table II. It should be emphasized that segmented linearization makes subjective assumptions over the location of the boundary points of different inactivation phases and that, as a result, errors made in demarcating the first inactivation phase are essentially passed onto the second and, if it exists, the third phases. This explains why, on the whole, all three inactivation models provide similar predictions of the first decimal reduction value, whereas those for the second and subsequent values of $D$ were widely at variance.

The above discussion suggests that the segmented linearization technique is reasonable in its prediction of the first decimal reduction value but that doubt must attach to values given for $D_{2}$ and $D_{3}$ due to the accumulation of errors arising in the estimation of $D_{1}$ being transferred to both of these values. Interestingly, large errors induced in the prediction of $D_{2}$ and/or $D_{3}$ have been independently reported [12]. By contrast, the polynomial-based empirical technique enables mathematically rigorous and reliable predictions of $D_{2}$ and $D_{3}$ to be made. It should be noted, however, that the value of $t_{2}$ deduced from (5) and (8) can in principle be either negative or even complex, thus leading to nonphysical interpretations. In other words, $D_{2}$ and $D_{3}$ are not always physically reliable yardsticks for describing the second and third phases of the inactivation curves. This highlights the inherent difficulties in attempting to use a single parameter, namely, the decimal reduction value, as one that is capable of representing the nonlinear nature of the second and subsequent phases of the inactivation curves. It is our contention that this can only be achieved using properly constituted mathematical models, exemplified by the inactivation models featured here.

Although not mathematically rigorous, the technique of assigning a decimal reduction value to each apparent inactivation phase may be thought of as intuitive and providing for a qualitative, rather than quantitative, comparison of different plasma inactivation curves. As it is a simpler indicator than the mathematical formulas of (3), (7), and (8), it can nonetheless play a useful role when applied to a group of plasma inactivation studies having vastly different timescales of inactivation. This is true for the first inactivation phase, but not for any subsequent phase.

The difficulty of the empirical model in providing physically meaningful values of $D_{2}$ and $D_{3}$ highlights that its anticipated mathematical superiority over the Baranyi and Weibull models is unjustified. Adding to the comparison of all three models in terms of their $R^{2}$ value and their prediction of $D_{1}$ (see Tables I and II), the Baranyi model appears to be a good model overall in terms of both accuracy and mathematical simplicity. Equally important is its capability to correlate its parameters $\left(N_{0}, C_{c}(0)\right.$, and $\left.k_{\max }\right)$ to quantities that carry either physical or biological significance. The Baranyi model is therefore an appropriate model for microbial inactivation by nonthermal plasmas and as a base to unravel the physical and biological mechanisms in future microbial inactivation studies using nonthermal plasmas.

Although the three inactivation models are proposed as a reference tool relating one plasma inactivation study to another, their further development could potentially lead to a correlation of the model parameters to key biological properties and physical features of nonthermal plasmas. This would ultimately allow the model parameters to be used as simple and measurable indicators of underpinning plasma physics or/and bacteriology. While this ultimate benefit requires many substantial research programs in the future, links of the model parameters to microbiological parameters can already be suggested. In the case of the Baranyi model, the shoulder, the exponential decay, and the tail phases of a typical inverse-S-shaped inactivation curve are discussed in Section II-A. This links bacterial properties to the $D_{1}, D_{2}$, and $D_{3}$ parameters and, consequently, to the model parameters $N_{0}, k_{\max }$, and $C_{c}(0)$ through (4)-(6). The fact that all inactivation data exhibit a very short shoulder phase suggests that the initial threshold of bacterial resistance is relatively easy for species of nonthermal plasmas to overcome. This is illustrated in the model by the $k_{\max }$ parameter. After this, most bacteria are sufficiently damaged and susceptible for rapid inactivation by plasma species. Hence, the exponential decay phase follows. Similar case can also be made for the Weibull model.

It is also possible to relate model parameters to physical parameters of nonthermal plasmas, such as charged particles, free radicals, excited species, and electric field [18]. It is, however, interesting to note that the shapes of all studied inactivation curves for $B$. subtilis spores show a common inverse-S 
shape, although nonthermal plasmas considered are produced in different gases and at different gas pressures. Therefore, model parameters are likely to represent damages by potential plasma inactivation agents, most likely to be synergistic, rather than specific plasma agents. Future studies are therefore required to substantiate this.

\section{CONCLUSION}

Justification for linearizing selected segments of microbial inactivation curves obtained using nonthermal plasmas was shown to be associated largely with practical simplicity. Although this approach does have the advantage of yielding a single parameter to characterize plasma inactivation using relatively uncomplicated procedures, it has been shown to be reliable only for the first inactivation phase but not for subsequent phases. Since the essentially nonlinear microbial inactivation curve does not normally display distinct and unambiguous boundary points between different inactivation phases, its accurate description can ultimately only be achieved by recourse to more rigorous mathematical models. To this end, three inactivation models were proposed for characterizing nonthermal plasma inactivation, namely: 1) the Baranyi model; 2) the Weibull model; and 3) a third-order polynomial empirical model. For B. subtilis spores, these models were shown to reproduce experimental inactivation data reliably and faithfully. These mathematically accurate models are therefore essential for a quantitatively accurate comparison of different plasma inactivation curves already reported and for the design of future and improved plasma inactivation studies. The Baranyi model was recommended as the appropriate basis to unravel physical and biological mechanisms of plasma microbial inactivation. Previous attempts to correlate apparent phases in the process of microbial inactivation to the generation of certain plasma species have met with only partial success. However, what must be the ultimate objective of such studies of identifying those biological processes that are affected by the various plasma species currently remains unfulfilled. It is only when this has been achieved that there will be any justification for segmenting inactivation curves.

\section{REFERENCES}

[1] A. E. Sloan, "Gourmet and specialist food trends," Food Technol., vol. 58, no. 7, p. 26, Jul. 2004

[2] S. Sivapalasingam, C. R. Friedman, L. Cohen, and R. V. Tauxe, "Fresh produce: A growing cause of outbreaks of foodborne illness in the United States, 1973 through 1997," J. Food Prot., vol. 67, no. 10, pp. 2342-2353, Oct. 2004

[3] J. Raso and G. V. Barbosa-Canovas, "Nonthermal preservation of foods using combined processing techniques," Crit. Rev. Food Sci. Nutr., vol. 43, no. 3, pp. 265-285, May 2003.

[4] M. Vleugels, G. Shama, X. Deng, E. Greenacre, T. Brocklehurst, and M. G. Kong, "Atmospheric plasma inactivation of biofilm-forming bacteria for food safety control," IEEE Trans. Plasma Sci., vol. 33, no. 2, pp. 824-828, Apr. 2005.

[5] M. Moison, J. Barbeau, M. C. Crevier, J. Pelletier, N. Philip, and B. Saoudi, "Plasma sterilization: Methods and mechanisms," Pure Appl. Chem., vol. 74, no. 3, pp. 349-358, Mar. 2002.

[6] K. Kelley-Wintenberg, T. C. Montie, C. Brickman, J. R. Roth, A. K. Carr, K. Sorge, L. C. Wadsworth, and P. P. Y. Tsai, "Room temperature sterilization of surfaces and fabrics with a one atmosphere uniform glow discharge plasma," J. Ind. Microbiol. Biotech., vol. 20, no. 1, pp. 69-74, Jan. 1998.
[7] J. Baranyi, A. Jones, C. Walker, A. Kaloti, T. P. Rabinson, and B. M. Mackey, "A combined model for growth and subsequent thermal inactivation of Brochothrix thermosphacta," Appl. Environ. Microbiol., vol. 62, no. 3, pp. 1029-1035, Mar. 1996.

[8] E. J. Greenacre, T. F. Brocklehurst, C. R. Waspe, D. R. Wilson, and P. D. G. Wilson, "Salmonella enterica serovar typhimurium and Listeria monocytogenes acid tolerance response induced by organic acids at 20 degrees C: Optimization and modeling," Appl. Environ. Microbiol., vol. 69, no. 7, pp. 3945-3951, Jul. 2003.

[9] I. Albert and P. Mafart, "A modified Weibull model for bacterial inactivation," Int. J. Food Microbiol., vol. 100, no. 1-3, pp. 197-211, Apr. 2005.

[10] A. H. Geeraerd, C. H. Herremans, and J. F. van Impe, "Structural model requirements to describe microbial inactivation during a mild heat treatment," Int. J. Food Microbiol., vol. 59, no. 3, pp. 185-209, Sep. 2000.

[11] X. Deng, J. Shi, G. Shama, and M. G. Kong, "Effects of microbial loading and sporulation temperature on atmospheric plasma inactivation of Bacillus subtilis spores," Appl. Phys. Lett., vol. 87, no. 15, p. 153901, Oct. 2005.

[12] N. Philip, B. Saoudi, M. Crevier, M. Moisan, J. Barbeau, and J. Pelletier, "The respective roles of UV photons and oxygen atoms in plasma sterilization at reduced gas pressure: The case of $\mathrm{N}_{2}-\mathrm{O}_{2}$ mixtures," IEEE Trans. Plasma Sci., vol. 30, no. 4, pp. 1429-1436, Aug. 2002.

[13] S. Moreau, M. Moisan, M. Tabrizian, J. Barbeau, J. Pelletier, A. Ricard, and L'H. Yahia, "Using the flowing afterglow of a plasma to inactivate Bacillus subtilis spores: Influence of the operating conditions," J. Appl. Phys., vol. 88, no. 2, pp. 1166-1174, Jul. 2000.

[14] S. Hury, D. R. Vidal, F. Desor, J. Pelletier, and T. Lagarde, "A parametric study of the destruction of Bacillus spores in low pressure oxygen-based plasmas," Lett. Appl. Microbiol., vol. 26, no. 6, pp. 417-421, Jun. 1998.

[15] B. Ostle, K. V. Turner, C. R. Hicks, and G. W. McElrath, Engineering Statistics: The Industrial Experience, A. Kugushev, Ed. Belmont, CA: Duxbury Press, 1996, pp. 382-386.

[16] J. Baranyi and T. A. Roberts, "A dynamic approach to predicting bacterialgrowth in food," Int. J. Food Microbiol., vol. 23, no. 3/4, pp. 277-294, Nov. 1994.

[17] M. A. J. S. van Boekel, "On the use of the Weibull model to describe thermal inactivation of microbial vegetative cells," Int. J. Food Microbiol., vol. 74, no. 1/2, pp. 139-159, Mar. 2002.

[18] X. T. Deng, J. J. Shi, and M. G. Kong, "Physical mechanisms of inactivation of Bacilus subtilis spores using cold atmospheric plasmas," IEEE Trans. Plasma Sci., vol. 34, no. 4, Aug. 2006.

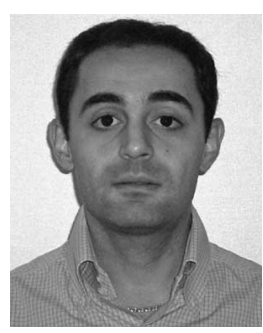

Stefano Perni received the M.Eng. degree in chemical engineering from the University of Bologna, Bologna, Italy, in 2001, and the Ph.D. degree from Loughborough University, Loughborough, U.K., in 2005 , for his work on biofilm formation.

Since 2005, he has been a Research Associate with the Department of Chemical Engineering, Loughborough University. His main research activity is focused on bacterial decontamination in food processing using cold plasmas and pulsed electric fields.

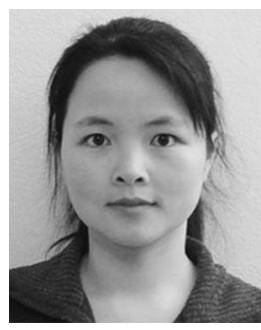

Xutao T. Deng received the B.Eng. degree in electronic engineering from Shenzhen University, Shenzhen, China, in 1999, the M.Sc.(Eng.) degree in intelligence engineering from the University of Liverpool, Liverpool, U.K., in 2000, and the Ph.D. degree in plasma engineering from Loughborough University, Loughborough, U.K., in 2004.

She is currently a Postdoctoral Research Scientist with the Plasma and Pulsed Power Group, Loughborough University. Her current research interests include protein reduction and bacterial inactivation using atmospheric-pressure glow discharges. 


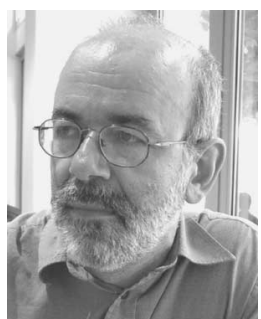

Gilbert Shama received the B.Sc. degree in chemical engineering from the University of Manchester, Manchester, U.K., in 1972, the M.Sc. degree in biological engineering from the University of Birmingham, Birmingham, U.K., in 1978, and the $\mathrm{Ph} . \mathrm{D}$. degree in microbial physiology from Imperial College, London, U.K., in 1985.

$\mathrm{He}$ is currently a Senior Lecturer with the Department of Chemical Engineering, Loughborough University, Loughborough, U.K. His interests include fermentation technology, photocatalysis, and microbial inactivation by various means and with particular relevance to the food industry.

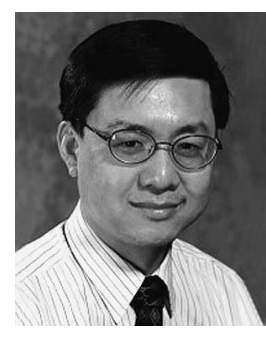

Michael G. Kong (M'94-SM'98) received the B.Sc. and M.Sc. degrees in electronics engineering from Zhejiang University, Hangzhou, China, in 1984 and 1987, respectively, and the Ph.D. degree in electrical engineering from Liverpool University, Liverpool, U.K., in 1992.

After his research and faculty positions with the Liverpool University and Nottingham University, U.K., he joined Loughborough University, Leicestershire, U.K., in 1999, where he holds a Chair in the bioelectrical engineering and leads the Plasma and Pulsed Power Group. At Loughborough University, he is also the Head of the Energy Research Division, whose research encompasses gas plasmas, pulsed power, and renewable energy. He has published approximately 150 papers in scientific journals and peer-reviewed conference proceedings. His current research interests include atmospheric-pressure glow discharges, ultrashort electric pulses, and their biomedical applications.

Dr. Kong is a Guest Editor for a special issue of the IEEE TRANSACTIONS ON PlASMA SCIENCE on the Nonthermal Medical/Biological Applications of Ionized Gases and Electromagnetic Fields (August 2006). 